\title{
Alineando el ciclo de vida de un proyecto con un modelo de madurez BI: una propuesta para la etapa de análisis preliminar
}

\author{
Aligning the life cycle of a project with a BI maturity model: \\ a proposal for the preliminary analysis stage
}

\author{
Loreto Castillo Abarca $^{1} \quad$ Vianca Vega Zepeda $^{1} \quad$ Claudio Meneses Villegas ${ }^{1 *}$ \\ Recibido 15 de julio de 2020, aceptado 1 de septiembre de 2020 \\ Received: July 15, 2020 Accepted: September 1, 2020
}

\begin{abstract}
RESUMEN
La Inteligencia de Negocios (BI por sus siglas en inglés) es una de las aplicaciones prácticas más relevantes de técnicas de análisis de datos y algoritmos de inteligencia artificial, que busca explotar en favor de una organización el activo que representan sus datos históricos internos y/o externos. Los proyectos de BI buscan aplicar estas tecnologías a la organización para obtener un beneficio. Sin embargo, los resultados de estos proyectos dependen grandemente de la madurez organizacional. Los modelos de madurez BI existentes ofrecen distintas visiones de la organización, con distintos niveles de detalles, estableciendo una dificultad para guiar el desarrollo de un proyecto de BI. Este trabajo persigue establecer un ciclo de vida de un proyecto de BI que se alinee con la madurez BI de una organización, de tal forma que las fases, actividades y tareas de este tipo de proyecto tengan relación con las indicadas por el modelo de madurez utilizado. Tras un proceso de selección de un modelo de madurez BI a utilizar por fase, se propone un ciclo de vida para un proyecto de BI, generando activos de proceso para las fases y actividades del mismo. En particular, dada la envergadura que representa el ciclo de vida de un proyecto de BI, este trabajo se limita a presentar detalles de esta guía para la primera fase del ciclo de vida definido, denominada Análisis Preliminar. Actualmente, este modelo de ciclo de vida de un proyecto BI está siendo aplicado a un caso real, después de lo cual se obtendrá datos para evaluar objetivamente su grado de completitud y utilidad práctica en el desarrollo de este tipo de proyecto.
\end{abstract}

Palabras clave: Inteligencia de negocios, modelo de madurez, ciclo de vida de un proyecto BI.

\begin{abstract}
Business Intelligence (BI) is one of the most relevant practical applications of data analysis techniques and artificial intelligence algorithms, which seeks to exploit in favor of an organization the asset that represents its internal historical data and / or external. BI projects seek to apply these technologies to the organization to obtain a benefit. However, the results of these projects depend greatly on organizational maturity. The existing BI maturity models offer different views of the organization, with different levels of detail, establishing a difficulty in guiding the development of a BI project. This work aims to establish a life cycle of a BI project that is aligned with the BI maturity of an organization, so that the phases, activities and tasks of this type of project are related to those indicated by the maturity model used. After a process of selecting a BI maturity model to be used for each phase, a life cycle is proposed for a BI project, generating process assets for the phases and activities of the same. In particular, given the scale
\end{abstract}

\footnotetext{
1 Universidad Católica del Norte, Departamento de Ingeniería de Sistemas y Computación, Antofagasta, Chile. E-mail: 1castillo@ucn.cl; vvega@ucn.cl; cmeneses@ucn.cl

* Autor de correspondencia: cmeneses@ucn.cl
} 
that represents the life cycle of a BI project, this work is limited to presenting details of this guide for the first phase of the defined life cycle, called Preliminary Analysis. Currently, this model of the life cycle of a BI project is being applied to a real case, after which data will be obtained to objectively evaluate its degree of completeness and practical utility in the development of this type of projects.

Keywords: Business intelligence, maturity model, life cycle of a BI project.

\section{INTRODUCCIÓN}

Hoy en día y gracias al crecimiento de la tecnología y del software, las organizaciones logran almacenar grandes volúmenes de datos que, si son debidamente procesados, pueden llegar a constituir una importante fuente de información. Esta información puede formar la base de conocimiento que requiere una organización para la toma de decisiones de manera objetiva por parte de los directivos o de quienes tienen una participación clave. Una manera de mejorar el manejo de información para el logro de los objetivos de negocios es a través del uso de la Inteligencia de Negocios (BI por su sigla en inglés) [1]. Sin embargo, emprender en la implementación de una estrategia de BI puede resultar difícil, debido a que, por sobre todo, un proyecto de BI va ligado estrechamente al plan estratégico de la organización [2].

Cuando se tiene en mente el desarrollo de un proyecto, de inmediato se piensa en el presupuesto, el recurso humano, las horas que requerirán ser invertidas, el o los asesores expertos que apoyarán al desarrollo del mismo, los tiempos que se tienen para desarrollarlo y la fecha de finalización del mismo. Pero, ¿la organización está preparada para enfrentar esta implementación? Y, ¿qué significa estar preparado?, ¿bastará tener un presupuesto para invertir en el proyecto?, ¿bastará tener el apoyo de expertos en BI? En base a la información analizada en este trabajo, se pudo notar que muchos de los estudios relacionados con este tema, comprenden las particularidades que implica levantar un proyecto de BI, proponen una serie de actividades a realizar para poder llevar a cabo el desarrollo de un proyecto, desde la conformación del equipo, hasta la adquisición e implementación de tecnología; sin embargo, por muy robusto que sea el proyecto, no garantiza que sea bien aprovechado después, porque existen otros factores que impiden que esto suceda. Uno de ellos es la cultura organizacional. Cuando se plantea aquí las preguntas antes citadas, se refiere al cómo conocemos nuestra forma de trabajar, de nada sirve tener implantado un gran proyecto de BI si no se sacará el provecho que realmente puede entregar. Por lo tanto, el presente trabajo propone analizar esta fase preliminar, de tal forma, sea cual sea la implementación de tecnología BI que se utilice, se tenga conciencia antes de la manera de trabajar de la organización.

Para el desarrollo de este trabajo, se comenzó con la revisión y selección de los modelos de madurez disponibles, distinguiendo aquel modelo que contenga como concepto a medir los procesos y sus actividades asociadas a las etapas del desarrollo de un proyecto.

Luego de este análisis se propone el ciclo de vida de un proyecto de BI que profundiza en la etapa del análisis preliminar, que abarca los puntos citados anteriormente. Este análisis permite describir las actividades a realizar antes de comenzar con el desarrollo mismo del proyecto. Entrega además la documentación a utilizar y las directrices a seguir para completar cada actividad.

En este trabajo se aborda y profundiza una etapa no considerada en el ciclo de vida de un proyecto BI: el poder orientar a una organización a identificar la necesidad y poder incorporar la conciencia del estado interno de su cultura o forma de trabajo, materializado en actividades concretas a realizar, lo cual permite sentar las bases sólidas para el desarrollo de un proyecto de BI. Desde ese punto, la organización estará preparada para seguir el camino que mejor calce a sus posibilidades, entendiendo esto como desarrollar un proyecto de BI propio o adquirirlo externamente.

\section{TRABAJOS RELACIONADOS}

Para el desarrollo de este trabajo, se realizó una búsqueda y recopilación de información relacionada con los distintos conceptos que se emplearon para este objetivo: modelos de madurez, proyectos de BI, y metodologías de comparación. A continuación, se destacan los conceptos y trabajos más relevantes. 


\section{Modelo de Madurez BI}

Se dice que, si algo se puede medir, se puede gestionar [3]. Para este propósito nacen los modelos de madurez, que permiten medir las capacidades de una organización sustentado en el conocimiento de los procesos internos de la empresa. Creados inicialmente para aplicarlos a la calidad y capacidad de las empresas de desarrollo de software. En la actualidad, su área de aplicación se ha diversificado llegando a áreas como gestión del conocimiento, gestión del desempeño y Gestión de Datos, entre otros [4]. Los modelos desarrollados para el dominio de BI tienen como ventajas importantes la facilidad de comprensión y en la prestación de una herramienta para comparar diferentes empresas y/o partes de ella entre sí [5].

En términos generales, un modelo de madurez permite [6]:

- Evaluar el estado de crecimiento de una organización o proceso de negocio.

- Que las organizaciones transiten de forma progresiva a través de los niveles de madurez, que abarcan desde la inconsistencia de los procesos de negocio hasta el nivel óptimo de mejora continua de los procesos de negocio.

- Trazar claramente estrategias de mejoras para alcanzar los objetivos previstos.

- Identificar las áreas o factores donde la organización debe enfocarse para mejorar.

- Guiar programas de mejoras.

Entonces, un modelo de madurez es una herramienta que permite identificar, evaluar y explicar los procesos de una organización a través de una secuencia de niveles que determina la evolución en que se encuentra bajo un contexto BI [7].

En la bibliografía, se observan variados modelos de madurez para distintos dominios y que han ido evolucionando, ya que los autores suelen mejorarlos basados en las experiencias pasadas de otros autores. La Tabla 1 preparada a partir de [5-6, 8-9], muestra un resumen de algunos modelos de madurez y sus distintos enfoques.

\section{Caracterización y Comparación de Modelos de Madurez}

Como parte del desarrollo de este trabajo es encontrar un modelo de madurez base, se hizo necesario investigar formas y metodologías para la caracterización y comparación de modelos dentro de la literatura. Por ejemplo, la definición de algunos criterios de selección utilizados en [6] cuyos autores tienen como finalidad encontrar un modelo de madurez que sea idóneo para utilizar en Pymes. Ellos privilegiaron algunas características como se indican en la Tabla 2 que permite evidenciar las bondades de los modelos de madurez estudiados.

Con estos criterios, los autores generan una estadística donde analizan el porcentaje de cumplimiento de cada criterio, aspecto que consideran para luego seleccionar el modelo más idóneo para emplear en las Pymes. Esta comparación dio como resultado la selección de un modelo de madurez que tiene un campo de aplicación más amplio, cuya estructura y facilidad de uso son amigables sin la necesidad de requerir un equipo experto para poder aplicarlo. Se notó, además, que seis de los siete modelos estudiados no disponen de procedimientos ni de herramientas de evaluación, aspectos que deben ser considerados para seleccionar el modelo de madurez para aplicarlo en las Pymes.

La siguiente referencia, corresponde a un estudio comparativo de estándares ISO y CMMI, donde los autores definen criterios de comparación para poder establecer semejanzas y diferencias para facilitar la selección del modelo o estándar que se ajusta mejor a las características de la organización. Los criterios establecidos se muestran en la Tabla 3 [10].

Finalmente, para realizar una comparación, los autores sugieren establecer parámetros que se van seleccionando de los distintos criterios establecidos en la tabla anterior, esto con el fin de establecer semejanzas y diferencias entre los modelos a comparar. Con cada uno de los criterios los autores realizan un mapeo modelo a modelo.

Otro tipo de análisis comparativo es la adaptación del modelo matemático DEA (Análisis envolvente de datos) para caracterizar los niveles de los modelos de madurez y medir su eficiencia. Como eficiencia, se entiende la capacidad cuantitativa de cada modelo analizado en una fase determinada para transformar las entradas en salidas. Contabiliza los elementos de entrada y salida en cada nivel de madurez.

DEA es una técnica muy robusta de optimización y corresponde a una de las herramientas centrales disponibles para el análisis de los objetivos de la 
Tabla 1. Resumen Modelos de Madurez para BI.

\begin{tabular}{|c|c|c|c|}
\hline Modelo & Aspectos & Niveles & Observaciones \\
\hline $\begin{array}{l}\text { Modelo de madurez } \\
\text { de TDWI }\end{array}$ & $\begin{array}{l}\text { - Alcance } \\
\text { - Patrocinio } \\
\text { - Financiamiento } \\
\text { - Valor } \\
\text { - Arquitectura } \\
\text { - Datos } \\
\text { - Desarrollo } \\
\text { - Entrega } \\
\end{array}$ & $\begin{array}{l}\text { - N.1: Lactante } \\
\text { - N.2: Niño } \\
\text { - N.3: Adolescente } \\
\text { - N.4: Adulto } \\
\text { - N.5: Sabio }\end{array}$ & $\begin{array}{l}\text { - La herramienta de evaluación y la documentación } \\
\text { está disponible en la Web. } \\
\text { - Se concentra en puntos de vista técnicos, y en } \\
\text { especial en el almacenamiento de datos. } \\
\text { - Se puede mejorar el punto de vista de negocio, } \\
\text { especialmente en el cultural y organizacional. }\end{array}$ \\
\hline $\begin{array}{l}\text { Madurez Jerárquica } \\
\text { de Inteligencia } \\
\text { de Negocios (BI } \\
\text { Maturity Hierarchy) }\end{array}$ & - Conocimiento & $\begin{array}{l}\text { - N.1: Datos } \\
\text { - N.2: Información } \\
\text { - N.3: Conocimiento } \\
\text { - N.4: Sabiduría }\end{array}$ & $\begin{array}{l}\text { - Los niveles de madurez están construidos desde } \\
\text { el punto de vista técnico. } \\
\text { - La documentación de este modelo no es suficiente } \\
\text { para la evaluación del nivel de madurez. }\end{array}$ \\
\hline $\begin{array}{l}\text { Modelo Enterprise } \\
\text { Intelligence }\end{array}$ & $\begin{array}{l}\text { - Datos } \\
\text { - Procesos } \\
\text { - Sistemas }\end{array}$ & $\begin{array}{l}\text { - N.1: Administración } \\
\text { - N.2: Identificación } \\
\text { - N.3: Preparación } \\
\text { - N.4: Utilización } \\
\text { - N.5: Apalancamiento }\end{array}$ & $\begin{array}{l}\text { - Abarca análisis de datos, sistemas y procesos, } \\
\text { además de arquitectura y gestión de conocimiento. }\end{array}$ \\
\hline $\begin{array}{l}\text { Modelo de madurez } \\
\text { de Gartner }\end{array}$ & $\begin{array}{l}\text { - Personas } \\
\text { - Habilidades } \\
\text { - Procesos } \\
\text { - Tecnología }\end{array}$ & $\begin{array}{l}\text { - N.1: Desconocido } \\
\text { - N.2: Oportunista } \\
\text { - N.3: Estándares } \\
\text { - N.4: Empresa } \\
\text { - N.5: Transformativo }\end{array}$ & $\begin{array}{l}\text { - Usos para evaluar los niveles de madurez de negocios } \\
\text { y la madurez de los distintos departamentos. } \\
\text { - Proporciona visión más técnica y no se concentra } \\
\text { en el aspecto técnico del negocio. } \\
\text { - Bien documentado y disponible en la Web. }\end{array}$ \\
\hline $\begin{array}{l}\text { Modelo de Madurez } \\
\text { de Información de } \\
\text { Negocios }\end{array}$ & - Cultura Empresarial & $\begin{array}{l}\text { - N.1: "Qué" } \\
\text { - N.2: "Quién, Cuándo, Dónde" } \\
\text { - N.3: "Cómo" }\end{array}$ & $\begin{array}{l}\text { - Cuestionario de autoevaluación bien documentado. } \\
\text { - Los criterios para evaluar el nivel de madurez } \\
\text { no están bien definidos. }\end{array}$ \\
\hline $\begin{array}{l}\text { Rendimiento de } \\
\text { la Inteligencia de } \\
\text { Negocios /AMR }\end{array}$ & $\begin{array}{l}\text { - Área de gestión y } \\
\text { desempeño }\end{array}$ & $\begin{array}{l}\text { - N.1: Como reacción } \\
\text { - N.2: Anticipando } \\
\text { - N.3: Colaborar } \\
\text { - N.4: Articular }\end{array}$ & $\begin{array}{l}\text { - Se concentra en la gestión del rendimiento. } \\
\text { - Poco documentado. } \\
\text { - Los criterios para evaluar el nivel de madurez } \\
\text { no están bien definidos. } \\
\text { - Sin cuestionario para evaluar los niveles de madurez. }\end{array}$ \\
\hline $\begin{array}{l}\text { Modelo de Madurez } \\
\text { de la Optimización } \\
\text { de la Infraestructura }\end{array}$ & \begin{tabular}{|l} 
- Eficacia en la \\
presentación de \\
informes \\
- Eficacia en el análisis \\
\end{tabular} & $\begin{array}{l}\text { - N.1: Básico } \\
\text { - N.2: Estandarizado } \\
\text { - N.3: Avanzado } \\
\text { - N.4: Dinámico } \\
\end{array}$ & $\begin{array}{l}\text { - Se centra en los productos y tecnologías en } \\
\text { lugar del punto de vista empresarial. } \\
\text { - Los criterios para la evaluación no están bien } \\
\text { definidos. }\end{array}$ \\
\hline $\begin{array}{l}\text { Escala de } \\
\text { inteligencia de } \\
\text { negocios (LOBI) }\end{array}$ & $\begin{array}{l}\text { - Tecnología } \\
\text { - Procesos } \\
\text { - Personas }\end{array}$ & $\begin{array}{l}\text { - N.1: Hechos } \\
\text { - N.2: Datos } \\
\text { - N.3: Información } \\
\text { - N.4: Conocimiento } \\
\text { - N.5: Entendimiento } \\
\text { - N.6: Intuición }\end{array}$ & $\begin{array}{l}\text { - Aplicado a la gestión del conocimiento. } \\
\text { - El autor construye niveles de madurez desde } \\
\text { el punto de vista técnico. } \\
\text { - Los criterios para evaluar el nivel de madurez } \\
\text { no están bien definidas. }\end{array}$ \\
\hline
\end{tabular}

Fuente: [5-6, 8-9].

Tabla 2. Características para seleccionar modelos de madurez BI para Pymes.

\begin{tabular}{|c|c|c|c|c|c|c|}
\hline Descripción del Modelo & Estructura & $\begin{array}{l}\text { ¿Fácil } \\
\text { de } \\
\text { usar? }\end{array}$ & $\begin{array}{l}\text { Procedimiento de } \\
\text { Aplicación }\end{array}$ & $\begin{array}{l}\text { Herramientas de } \\
\text { Evaluación }\end{array}$ & $\begin{array}{l}\text { Alcance de } \\
\text { Evaluación }\end{array}$ & $\begin{array}{l}\text { Campo de } \\
\text { Aplicación }\end{array}$ \\
\hline $\begin{array}{l}\text { - Cantidad de estados de } \\
\text { madurez. } \\
\text { - Factores críticos. } \\
\text { - Factores claves de éxito. } \\
\text { - Contiene Objetivos. } \\
\text { - Contiene prácticas. } \\
\text { - Facilitadores de Procesos. }\end{array}$ & $\begin{array}{l}\text { - Simple. } \\
\text { - Compleja. } \\
\text { - Algún } \\
\text { grado de } \\
\text { complejidad. }\end{array}$ & $\begin{array}{l}-\mathrm{Si} \\
\text { - No. }\end{array}$ & $\begin{array}{l}\text { - No existe. } \\
\text { - Disponible y } \\
\text { complejo. } \\
\text { - No está disponible. }\end{array}$ & $\begin{array}{l}\text { - Disponible (Matriz } \\
\text { de evaluación, } \\
\text { modelo genérico). } \\
\text { - No disponible }\end{array}$ & $\begin{array}{l}\text { - Organización. } \\
\text { - Procesos. } \\
\text { - Procesos y } \\
\text { Organización. }\end{array}$ & $\begin{array}{l}\text { - Cualquier } \\
\text { ámbito. } \\
\text { - Software. } \\
\text { - BPM. }\end{array}$ \\
\hline
\end{tabular}

Fuente: [6]. 
Tabla 3. Criterios y Parámetros de comparación para modelos y estándares.

\begin{tabular}{|c|c|c|}
\hline Criterio & Parámetro & Observación \\
\hline \multirow{3}{*}{$\begin{array}{l}\text { Alcance del } \\
\text { modelo o } \\
\text { estándar }\end{array}$} & Ámbito de la aplicación & Indica si es una mejora o para determinar su capacidad \\
\hline & Objetivos del modelo o estándar & Indica si será utilizado para una mejora o con fines de evaluación \\
\hline & Enfoque del modelo o estándar & \\
\hline \multirow{6}{*}{ Arquitectura } & Tipo de Framework & $\begin{array}{l}\text { Indica si es un modelo o un estándar. Para este trabajo, sería } \\
\text { un modelo. }\end{array}$ \\
\hline & Tipo de representación & Indica si es continuo o en etapas \\
\hline & Estructura & \\
\hline & Método para la mejora de procesos & \\
\hline & Categoría de procesos & \\
\hline & Escala de medición del mejoramiento & \\
\hline \multirow{4}{*}{ Funcionalidad } & Validación del modelo estándar & $\begin{array}{l}\text { Para determinar los mecanismos de validación del modelo } \\
\text { o estándar }\end{array}$ \\
\hline & Técnicas de análisis & \\
\hline & Tiempo de implantación & \\
\hline & Modelo del ciclo de vida & \\
\hline \multirow{5}{*}{$\begin{array}{l}\text { Características } \\
\text { de la } \\
\text { organización }\end{array}$} & Tamaño de la organización & $\begin{array}{l}\text { Indica si el modelo es aplicable a pequeñas, medianas o } \\
\text { grandes organizaciones }\end{array}$ \\
\hline & Cultura organizacional & $\begin{array}{l}\text { Indica los recursos que se necesitan para implantar el } \\
\text { modelo o estándar }\end{array}$ \\
\hline & Estructura de la organización & \\
\hline & Recursos para la implantación del modelo o estándar & \\
\hline & $\begin{array}{l}\text { Personal requerido para la implantación del modelo } \\
\text { estándar }\end{array}$ & Indica si se requiere de personal extra o externo a la organización \\
\hline
\end{tabular}

Fuente: [10].

organización. Esta técnica se ha aplicado a distintos rubros de organizaciones interesadas en medir la eficiencia.

Esta herramienta fue utilizada por Prieto [11] con el fin de realizar una comparación cuantitativa de un conjunto de modelos de madurez objetos de análisis. El autor adaptó la metodología DEA para analizar la eficiencia de los distintos estados de un conjunto de modelos de madurez de BI. La clave de esta adaptación es la identificación de los elementos necesarios para realizar la estimación de eficiencia.

Como primera actividad se identifican las unidades de decisión o MDU, que deben ser entidades donde se tomen decisiones, donde se transforman insumos en productos y servicios. En este sentido, se asoció cada Nivel de madurez como un MDU.

Como segunda actividad, se definió los inputs como cada actividad, prácticas y/o herramientas de software de BI descritas en cada nivel. Para los outputs se definió las KPA o áreas de proceso clave relacionadas en cada nivel (Ver Figura 1).
Establece además una eficiencia relativa que permite cuantificar en término de unidades los criterios definidos para cada uno de los modelos de madurez analizados.

La fórmula de la eficiencia relativa viene dada por:

$$
\text { Eficiencia relativa }=\frac{\text { Output } * \text { PesoOutput }}{\text { Input } * \text { PesoInput }}
$$

Los criterios a utilizar como input son:

- Cada una de las actividades o prácticas descritas en cada nivel.

- Cada una de las herramientas o software de BI descritas en cada nivel.

- Cada una de las metodologías descritas en cada nivel.

Los criterios a utilizar como output son:

- Cada una de las KPA pertenecientes al nivel.

- Cada una de las dimensiones pertenecientes al nivel (en caso de no haber KPA). 
Fuente: [11].

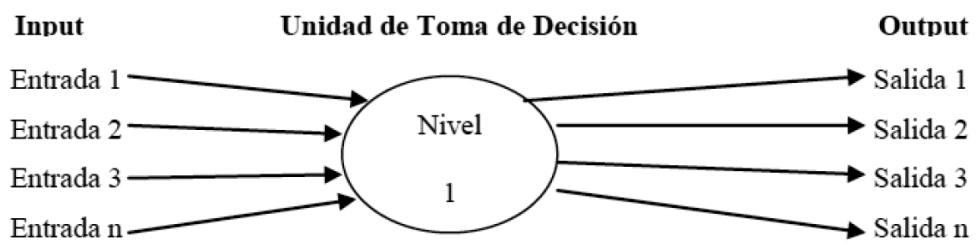

Figura 1. MDU, Inputs y Outputs del Nivel 1.

De lo anterior expuesto, el autor infiere que los modelos que contengan la misma cantidad de entradas y salidas tendrán el 100\% de eficiencia relativa, y también observa que estos modelos mantienen la cantidad de entradas y salidas a lo largo de todos sus niveles.

La aplicación de este modelo matemático permite cuantificar el máximo posible de eficiencia relativa en cada uno de los niveles de madurez, del conjunto de modelos, pero no permite ser concluyente a la hora de elegir un modelo en específico, salvo que este sea el único criterio que se necesite para la selección.

\section{Ciclo de Vida de un Proyecto de BI}

Un proyecto de BI tiene como objetivo básico dotar a una organización de los medios necesarios para que pueda tomar las decisiones estratégicas y operacionales adecuadas tomando como base el análisis de datos [12].

Los proyectos de BI requieren de conocimiento técnico del origen de los datos y qué quieren decir, además de la construcción de una arquitectura intermedia, que debe ser permanente y flexible. Sin embargo, más allá del conocimiento tecnológico de la implementación de una solución de BI, los proyectos de esta naturaleza poseen algunos puntos a considerar [13]:

- Enfoque en las necesidades del negocio, las personas y los procesos, por sobre la tecnología.

- El patrocinio directivo en todo momento.

- La capacidad de estructurar una arquitectura que sea capaz de sostenerse en el tiempo.

- Capacidad de dar respuesta rápida a los directivos.

- Obtener datos de alta calidad.

- Transversal a toda la organización a diferencia de un sistema transaccional convencional.

- La construcción de un sistema de inteligencia de negocio es mucho más lenta y no necesariamente muestra resultados visibles de inmediato por lo que requiere de la confianza y patrocinio de los ejecutivos claves

- Un proyecto BI debe estar preparado para los cambios en la estrategia del negocio.

Un esfuerzo de BI adecuadamente enfocado tiene el potencial de generar un profundo cambio cultural en la empresa, haciendo cada vez más natural la toma de decisiones basada en hechos y en información, rompiendo las barreras generadas por el control de la información [14]. De ahí que llevar a cabo un proyecto de BI sea diferente y muchas veces catalogado de difícil de implementar por poseer características que lo hacen único, tal como Rodríguez [13] plantea cuatro características cualitativas y que no posee otro tipo de proyecto:

El dueño del proyecto viene siendo el negocio, no típicamente un departamento de TI.

- Son ampliamente integradores y transversales debido a que la información la genera y necesita la mayoría de las personas claves de la organización.

- Su implementación va de la mano también con la alta tecnología específica para el almacenamiento, transformación y el análisis de datos.

- $\mathrm{Al}$ ser un proyecto que afecta transversalmente a la organización requiere de mucha capacidad de liderazgo y trabajo en equipo.

Como también es parte del desarrollo de este trabajo proponer un ciclo de vida de un proyecto de BI, es que se hace necesario revisar algunas propuestas de diferentes autores.

El ciclo de vida de un proyecto es una secuencia de fases que conectan el inicio con el fin de un proyecto. Permiten definir qué trabajo técnico se debe realizar en cada fase; cuándo se deben generar 
los productos entregables; quién está involucrado en cada fase y cómo controlar y aprobar cada una de ellas [15].

La guía de Fundamentos para la dirección de proyectos PMBOK [16], es un aporte reconocido, debido a que las prácticas y conocimientos descritos en ella son aplicable a la mayoría de los proyectos, representa una base sobre la que se puede construir metodologías, políticas, procedimientos, reglas, herramientas, técnicas y fases del ciclo de vida, necesarios para la práctica de la dirección de proyectos. Esta guía define un Proyecto, como un esfuerzo temporal que se lleva a cabo para crear un producto, servicio o resultado único.

La guía PMBOK es muy extensa, sin embargo, para el desarrollo de este trabajo, interesa conocer los componentes claves que son presentados en la Tabla 4 y Figura 2, que permitirá comprender la estructura general y de qué modo poder adaptarlo a la propuesta.

Con respecto a proyectos directamente relacionados con BI, se encuentra el trabajo de Buitrago [17], quien propone una metodología de desarrollo de proyectos BI basada en la disciplina de la gestión del alcance, debido a que las tareas que implican la ejecución del proyecto, son organizadas bajo una estructura Work break down o EDT, es decir, estructura de descomposición del trabajo. Gestión del tiempo, porque se determinan las actividades
Fuente: [16].

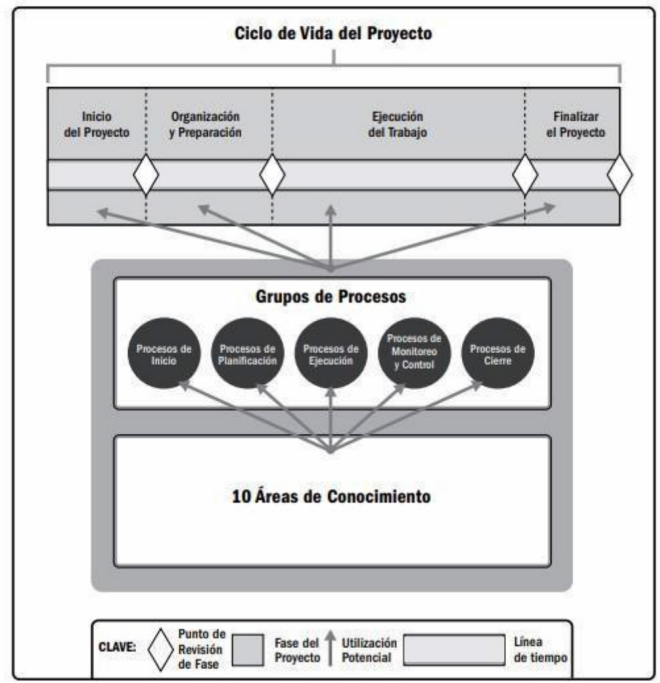

Figura 2. Interrelación entre los componentes Clave de los Proyectos de la Guía del PMBOK.

y la secuencia de actividades a realizar. Gestión de recursos humanos, porque se define el equipo de trabajo, roles, recursos, funciones y habilidades (basados en PMBOK).

La metodología comprende las siguientes fases:

1. Definición: Es el inicio del proyecto, donde se identifican todos los involucrados. También es donde se define el plan de trabajo.

Tabla 4. Descripción de los componentes Clave de la Guía PMBOK.

\begin{tabular}{|l|l|}
\hline $\begin{array}{c}\text { Componentes Claves de } \\
\text { la Guía PMBOK }\end{array}$ & \multicolumn{1}{c|}{ Breve Descripción } \\
\hline Ciclo de Vida del Proyecto & Serie de fases que atraviesa un proyecto desde su inicio hasta su conclusión. \\
\hline Fase del Proyecto & $\begin{array}{l}\text { Conjunto de actividades del proyecto relacionadas lógicamente que culmina con } \\
\text { la finalización de uno o más entregables. }\end{array}$ \\
\hline Punto de Revisión de Fase & $\begin{array}{l}\text { Revisión al final de una fase en la que se toma una decisión de continuar a la siguiente } \\
\text { fase, continuar con modificaciones o dar por concluido un programa o proyecto. }\end{array}$ \\
\hline $\begin{array}{l}\text { Procesos de la dirección } \\
\text { de proyectos }\end{array}$ & $\begin{array}{l}\text { Serie sistemática de actividades dirigidas a producir un resultado final de forma tal } \\
\text { que se actuará sobre una o más entradas para crear una o más salidas. }\end{array}$ \\
\hline $\begin{array}{l}\text { Grupo de procesos de la } \\
\text { dirección de proyectos }\end{array}$ & $\begin{array}{l}\text { Agrupamiento lógico de las entradas, herramientas, técnicas y salidas relacionadas } \\
\text { con la dirección de proyectos. Incluyen procesos de inicio, planificación, ejecución, } \\
\text { monitoreo y control, y cierre. }\end{array}$ \\
\hline $\begin{array}{l}\text { Área de conocimiento de } \\
\text { la dirección de proyectos }\end{array}$ & $\begin{array}{l}\text { Área identificada de la dirección de proyectos definida por sus requisitos de } \\
\text { conocimientos y que se describe en términos de sus procesos, prácticas, datos } \\
\text { iniciales, resultados, herramientas y técnicas que los componen. }\end{array}$ \\
\hline
\end{tabular}

Fuente: [16]. 
2. Levantamiento de Información: Esta fase se caracteriza por la búsqueda de la información necesaria para la construcción de la plataforma. Se definen las áreas de negocio y los productos que se desean obtener. También se definen los requerimientos por áreas y, por tanto, se definen los responsables de cada una.

3. Diseño: Corresponde al diseño de la plataforma. Se deben tomar en cuenta las herramientas de software requeridas. En esta etapa se realiza el modelado del negocio en base a la etapa anterior.

4. Desarrollo: En esta fase se construyen los diferentes componentes de la plataforma y productos definidos en la etapa anterior. En esta etapa también se construye el Data Warehouse y los procedimientos de extracción y carga.

5. Pruebas: En esta fase se verifica que tanto la funcionalidad como la información disponible estén de acuerdo con las especificaciones iniciales del proyecto.

6. Entrenamiento: Corresponde al traspaso de la información a todos los involucrados incluyendo a los usuarios finales. Se debe incluir el plan de entrenamiento.

7. Cierre: Corresponde al cierre de la etapa del proyecto. Debe contemplar la entrega de aceptación de la etapa y la entrega de documentación.

Cada una de las fases mencionadas comprende tareas, recursos y productos (ver Figura 3).

Otra propuesta de ciclo de vida de un proyecto e BI es el de los autores Larissa Moss y Shaku Atre [18], quienes proponen una guía paso a paso para el desarrollo de aplicaciones de soporte a decisiones de BI y cuyo propósito principal es explicar la complejidad de los proyectos de apoyo a la decisión y presentar la guía que identifica el ciclo de vida completo de un proyecto de BI. El esquema general que apoya esta propuesta se resume en la Figura 4.

El desarrollo de esta propuesta está definida por Fases $->$ Pasos $->$ Actividades $->$ Tareas o Subtareas.

Cada Fase corresponde a un capítulo, donde no tan solo se describen las actividades a realizar, sino también se adicionan los siguientes elementos:

- Resultado de las actividades.

- Roles involucrados en las actividades.

- Riesgos de no realizar la fase.

- Anexos como matrices de apoyo para cada fase.

\section{SELECCIÓN DE MODELOS DE MADUREZ BI}

\section{Proceso de Selección de un Modelo de Madurez Organizacional en BI}

En cuanto a la información recopilada en la sección de trabajos relacionados, específicamente a la relacionada con la comparación entre modelos de madurez, no fue posible utilizar el método cuantitativo DEA debido a que los modelos de madurez estudiados son muy diferentes entre sí, es decir, el ámbito al que hacen referencia no satisfacía la necedad del estudio, y por otro lado, la selección del modelo tiene una estrecha relación a la referencia que hace en un proyecto de $\mathrm{BI}$.

Como el objetivo de este trabajo, es proponer una etapa preliminar de un ciclo de vida, y que dentro de ella se pueda aplicar un modelo de madurez adecuado, es que se ideó la forma de selección de dicho modelo identificando aquel que contenga en su medición la mayor cantidad referencias hacia las

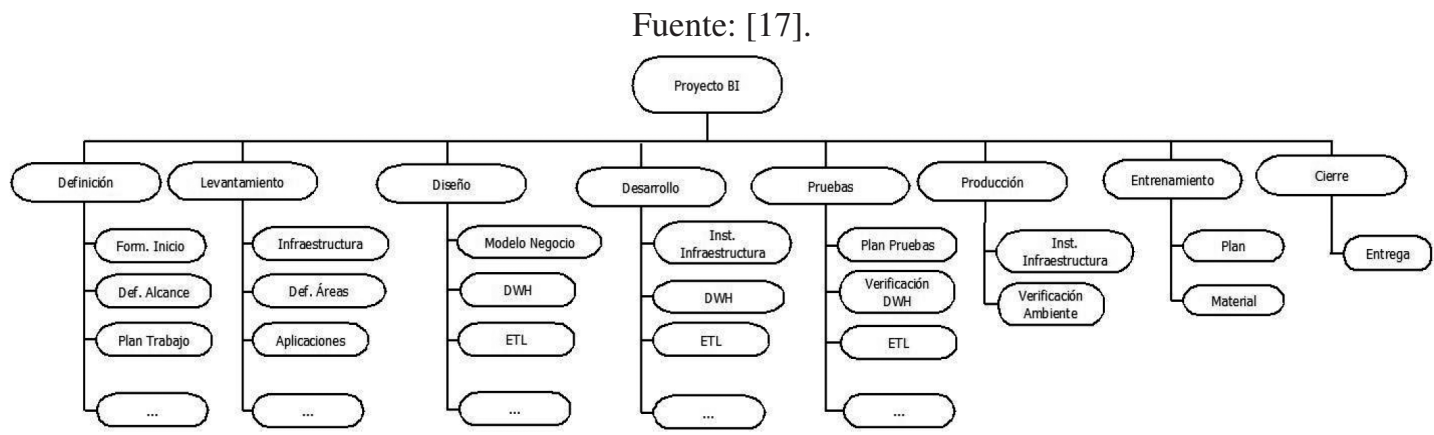

Figura 3. Resumen estructura de trabajo en función de las fases del proyecto de BI propuesto. 
Fuente: [17].

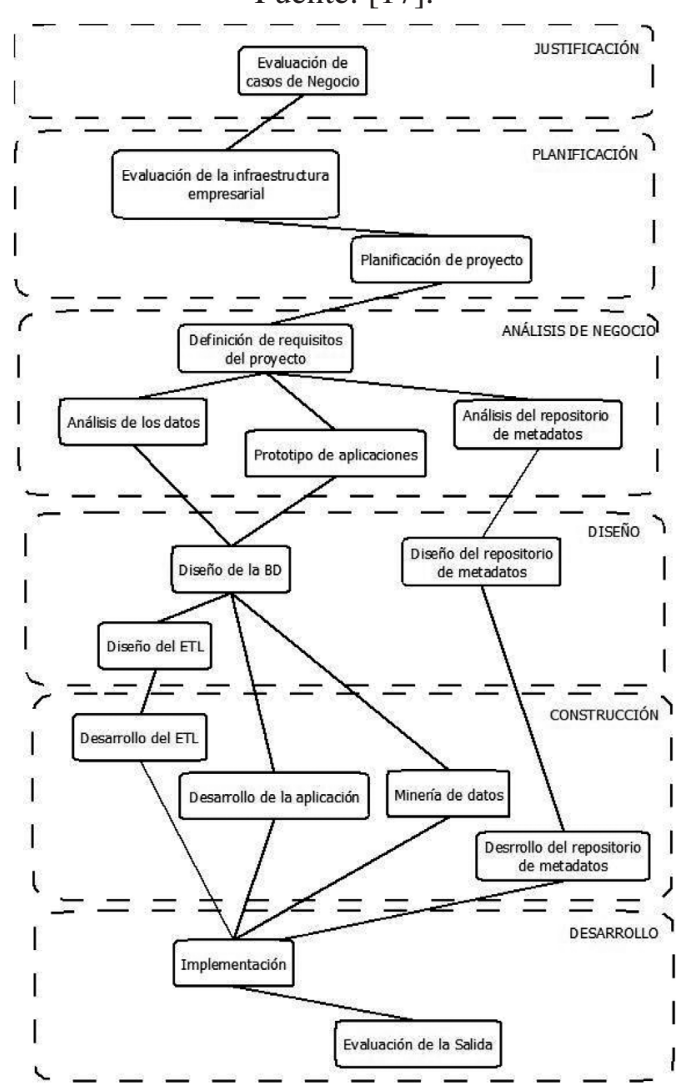

Figura 4. Esquema General del ciclo de vida propuesto por L. Moss y S. Atre. actividades iniciales de un proyecto de BI. Para esto se utiliza una metodología cualitativa, en donde se puedan alinear y caracterizar los modelos de madurez con un proyecto de $\mathrm{BI}$ base.

Para dar forma a esta idea, se crea un esquema que permite estructurar el desarrollo de la propuesta.

En primer lugar, se requiere seleccionar un modelo de madurez que permita alinearse con las actividades de un proyecto de BI. Para ello, es preciso caracterizar los modelos de madurez revisados según criterios de comparación. Luego, caracterizar los ciclos de vida de proyectos BI.

Con ambos alineados, se seleccionará el modelo de madurez que mejor se ajuste.

El esquema que representa esta primera sección se muestra en la Figura 5.

Para la caracterización del modelo de madurez se definieron dos criterios de selección.

1. Modelos cuya documentación estuviera disponible y de fácil acceso.

a. Modelo de Madurez de TDWI.

b. Modelo de Madurez Hierarchy.

c. Modelo Enterprise Intelligence (MEI).

Fuente: [Elaboración propia].

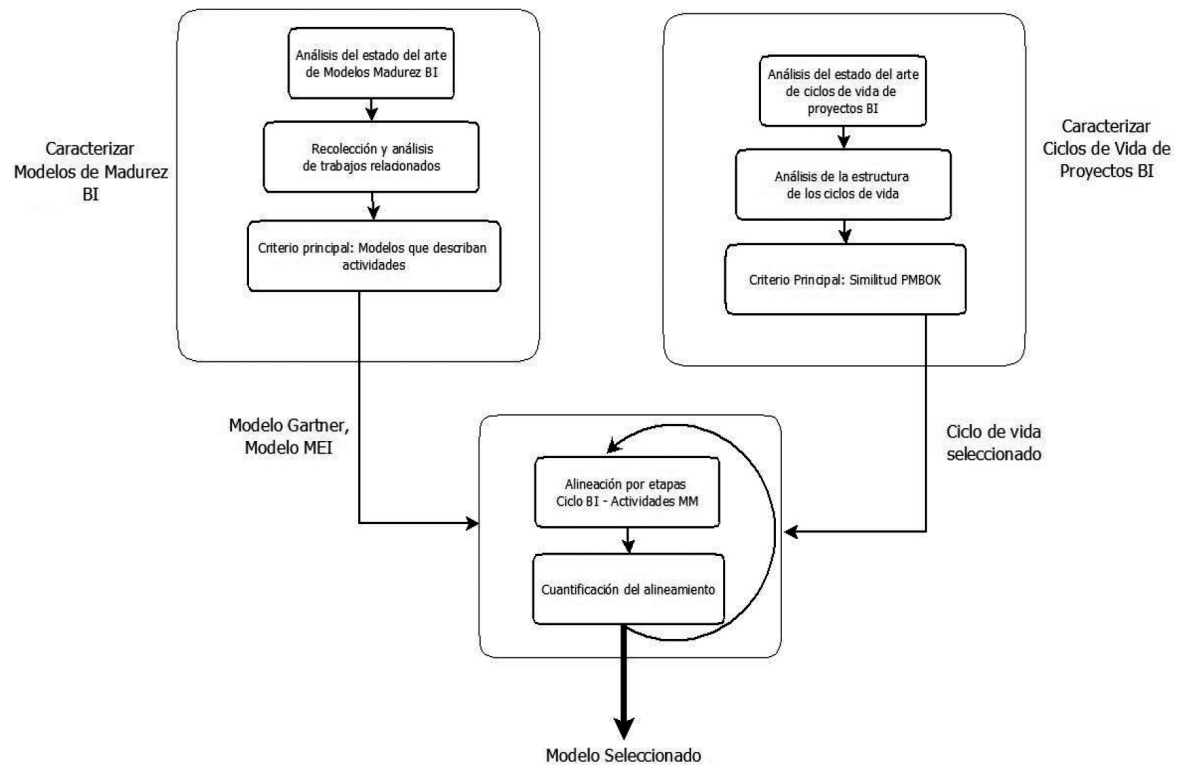

Figura 5. Esquema General Proceso de Selección Modelo Madurez. 
d. Modelo de Madurez de Gartner.

2. Modelos que en su estructura definieran actividades por nivel.

a. Modelo Enterprise Intelligence (MEI).

b. Modelo de Madurez de Gartner.

Este último criterio nos permitirá alinearlo con las actividades descritas en el ciclo de vida de proyecto BI elegido como base, con el fin de poder determinar actividades concretas para un proyecto de BI.

Para la caracterización del ciclo de vida de un proyecto de BI, se definió como criterio primordial, que esté alineado con el modelo establecido por PMBOK.

El modelo elegido es la adaptación de Buitrago [17].
Para esto, se dispuso en una matriz las fases del proyecto de BI con sus actividades y en las columnas siguientes, la correspondiente actividad de cada modelo de madurez (Tabla 5). Para facilitar la visualización, se les asignó un identificador a cada fase y actividad tanto del proyecto de BI como de los modelos de madurez.

Por ejemplo, para la Fase 1 (F1) del proyecto de BI llamado DEFINICIÓN, se buscó qué actividad del modelo de madurez corresponde a la del modelo de madurez ("A.n" para Gartner y "B.n” para MEI).

Luego, se cuantifica la cantidad de actividades del modelo de madurez presentes en la fase inicial del proyecto (se da prioridad a esta etapa porque es la que nos convoca en esta propuesta).

Tabla 5. Ejemplo de alineación de las actividades de la Fase Definición con las actividades de los modelos de Madurez preseleccionados.

\begin{tabular}{|c|c|c|c|c|c|c|c|c|}
\hline \multicolumn{3}{|c|}{ Proyecto BI } & \multicolumn{3}{|c|}{ A. Modelo de Madurez de Gartner } & \multicolumn{3}{|c|}{ B. Model Enterprise Intelligence (EI) } \\
\hline Fases & \multicolumn{2}{|r|}{ Actividades } & \multirow{2}{*}{$\frac{\text { Nivel }}{1}$} & \multicolumn{2}{|r|}{ Actividades } & \multirow[t]{2}{*}{ Nivel } & \multicolumn{2}{|r|}{ Actividades } \\
\hline \multirow{10}{*}{$\vec{x}$} & F1.1 & \begin{tabular}{|l|} 
Formalización \\
Inicio
\end{tabular} & & A.1 & Identificar los impulsores del negocio & & & \\
\hline & & & 1 & A. 2 & Obtener compromiso y recursos & & & \\
\hline & & & 2 & A. 4 & $\begin{array}{l}\text { Obtener financiación para iniciativas de BI } \\
\text { de altos ejecutivos }\end{array}$ & & & \\
\hline & F1.2 & $\begin{array}{l}\text { Definición } \\
\text { Alcance }\end{array}$ & 2 & A.3 & $\begin{array}{l}\text { Comprender los orígenes y calidad de los } \\
\text { datos, arquitectura y sistemas }\end{array}$ & & & \\
\hline & & & 2 & A.7 & $\begin{array}{l}\text { Necesidad de un centro de inteligencia de } \\
\text { negocios, donde expertos del negocio y de TI } \\
\text { se unan a fin de cumplir con las necesidades } \\
\text { del usuario. }\end{array}$ & & & \\
\hline & F1.3 & $\begin{array}{l}\text { Formación } \\
\text { Equipo }\end{array}$ & & & & & & \\
\hline & & & & & & & & \\
\hline & F1.4 & Definición plan & & & & 1 & B. 12 & $\begin{array}{l}\text { Supervisar todas las tareas de } \\
\text { gestión de cambios asociados con } \\
\text { las diversas áreas de proceso clave } \\
\text { para la inteligencia empresarial. }\end{array}$ \\
\hline & & & & & & 1 & B. 13 & $\begin{array}{l}\text { Definir procesos para aprovechar } \\
\text { los recursos dentro de la base de } \\
\text { conocimiento de la empresa y de } \\
\text { las agencias externas de apoyo } \\
\text { a las decisiones de la empresa }\end{array}$ \\
\hline & & & & & & 1 & B.14 & $\begin{array}{l}\text { Establecer métodos de comu- } \\
\text { nicación para simplificar el acceso } \\
\text { a la información relacionada con } \\
\text { las áreas de proceso clave para la } \\
\text { inteligencia empresarial. }\end{array}$ \\
\hline
\end{tabular}

Fuente: Elaboración Propia. 
El resumen se muestra en la Tabla 6.

Si bien existe una mayor cantidad de actividades descritas para el modelo MEI, las actividades del modelo de Gartner están presentes en las primeras actividades de la fase 1 (Tabla 5).

También, se puede notar en la Tabla 4, que las actividades del modelo de Gartner quedan consecutivas, es decir, existe una mejor alineación entre actividades (secuencia del 1 al 7). Sin embargo, para el modelo MEI, recién aparece en la actividad 12 de su modelo.

Es por ambas razones que para este propósito se elige el Modelo de Gartner.

\section{PROPUESTA DE UN CICLO DE VIDA DE UN PROYECTO DE BI}

Con todo lo anterior analizado, se propone un ciclo de vida formado por etapas, actividades específicas (que contienen a su vez sub-actividades) y actividades transversales que están presentes en todas las etapas. El esquema general se muestra en la Figura 6.

Del mismo modo, se propone fortalecer la etapa inicial del ciclo de vida de un proyecto de BI, incorporando una Etapa Preliminar.

El ciclo completo comprende entonces 6 etapas: Análisis Preliminar, Definición, Levantamiento de Información, Diseño, Implementación y Cierre de

Tabla 6. Resumen cuantificación actividades de modelo de madurez correspondientes a fases iniciales del proyecto de $\mathrm{BI}$.

\begin{tabular}{|l|l|c|c|}
\hline \multicolumn{1}{|c|}{$\begin{array}{c}\text { Fases } \\
\text { Proyecto BI }\end{array}$} & \multicolumn{1}{c|}{$\begin{array}{c}\text { Actividades } \\
\text { Proyecto BI }\end{array}$} & $\begin{array}{c}\text { Cantidad } \\
\text { Actividades Gartner }\end{array}$ & $\begin{array}{c}\text { Cantidad } \\
\text { Actividades MEI }\end{array}$ \\
\hline \multirow{3}{*}{$\begin{array}{l}\text { Fase1: } \\
\text { Definición }\end{array}$} & Formalización Inicio & 3 & 0 \\
\cline { 2 - 4 } & Definición Alcance & 2 & 0 \\
\cline { 2 - 4 } & Formación Equipo & 0 & 0 \\
\cline { 2 - 4 } & Definición plan & 0 & 3 \\
\hline \multirow{4}{*}{$\begin{array}{l}\text { Fase 2: } \\
\text { Levantamiento } \\
\text { de información }\end{array}$} & Identificación de áreas y procesos de negocio. & 4 & 7 \\
\cline { 2 - 4 } & Identificación de requerimientos de información. & 2 & 3 \\
\cline { 2 - 4 } & Identificación de aplicaciones operacionales & 0 & 3 \\
\cline { 2 - 4 } & Análisis de infraestructura tecnológica & 0 & 0 \\
\cline { 2 - 4 } & Identificación de requerimientos de la aplicación & 1 & 9 \\
\cline { 2 - 4 } & Identificación De requerimiento no funcionales & 1 & 0 \\
\hline Fase 3: Diseño & Diseño del modelo del negocio & 2 & \\
\hline
\end{tabular}

Fuente: Elaboración Propia.

Fuente: Elaboración propia.

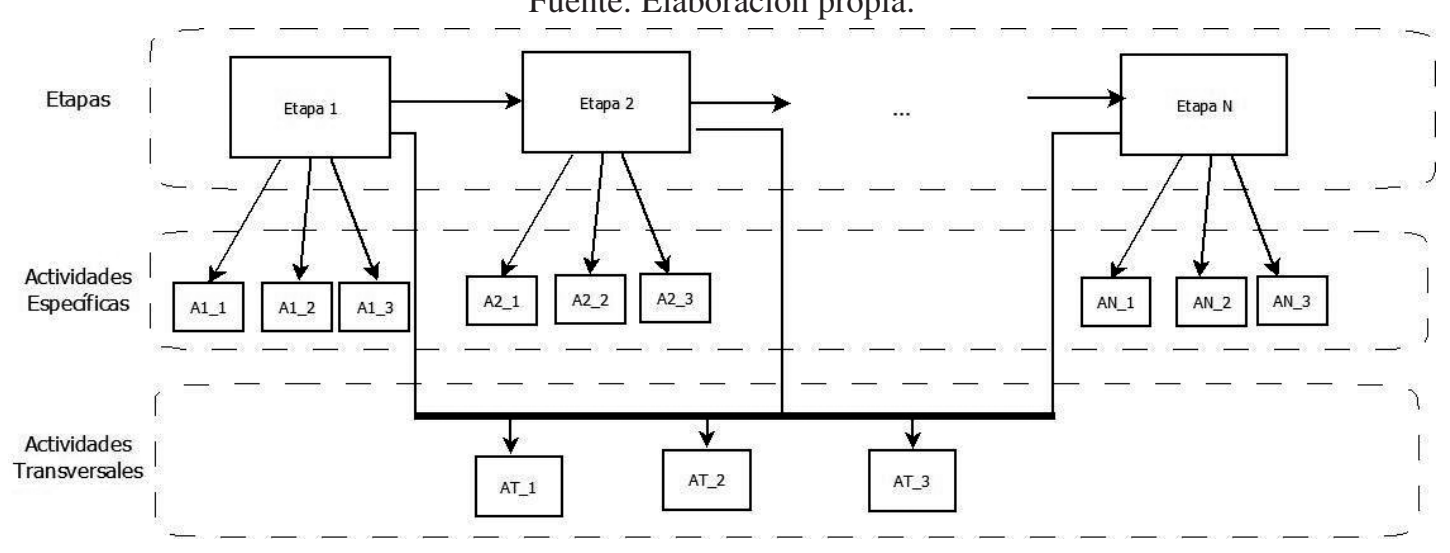

Figura 6. Esquema general del ciclo de vida de un proyecto de BI propuesto. 
Proyecto. Y acompañarán a cada etapa un conjunto de actividades llamadas transversales, cuyo dominio pertenece a: Gestión del Riesgo, Gestión del Cambio y Capacitación y Comunicación. El ciclo propuesto se muestra en la Figura 7.

Con mayor detalle, en la Figura 8, se presenta las actividades a realizar para la etapa de Análisis Preliminar, que permitirá comprender desde un enfoque organizacional el estado de la organización en cuanto a iniciativas de BI, así como alinear las necesidades del negocio con el plan estratégico organizacional, comprender las políticas internas y la cultura organizacional para el manejo de la información. También permite determinar los roles de todos los involucrados y finalmente, determinar los riesgos asociados a las actividades a realizar.

La Tabla 7 muestra las principales actividades, sub-actividades, objetivo y riesgos identificados para la etapa de Análisis Preliminar del ciclo de vida propuesto para un proyecto de $\mathrm{BI}$.

\section{Activos de Proceso}

La propuesta incluye también la formulación de activos de proceso para la generación de los resultados (salidas) de las actividades que componen cada etapa del ciclo de vida propuesto. Para ilustrar esto, en la Figura 9 se presenta a modo de ejemplo la Matriz Problema/Oportunidad correspondiente a la subactividad Análisis de Problema/Oportunidad. Se incluye también el objetivo de la correspondiente sub-actividad, que en este caso es comprender la situación actual, con el fin de poder determinar rutas de acción que respondan a las necesidades y crecimiento de la organización.
Para efectos del llenado de esta matriz, se debe considerar las siguientes definiciones para la clasificación de los problemas u oportunidades:

- Explícitos: Corresponden a causas claras.

- No Explícitos: corresponden a causas no claras.

- Respuesta inmediata: Se requiere una respuesta inmediata en la que solo queda actuar.

- Anticipación: plan hecho previamente, más provechoso.

- Investigación: Aparece mayormente cuando hay un emprendimiento nuevo o para una situación que resulta ajeno a lo cotidiano.

- Crisis/Oportunidad desperdiciada: No necesariamente es mala, puede llevar lidiar con imprevistos que finalmente resultan como aprendizajes valiosos.

- Urgente: requiere atención inmediatamente.

- No Urgente: No requiere de atención inmediata.

De la matriz Problema/Oportunidad, se obtienen además los siguientes componentes:

- Las situaciones que se ubican en el cuadrante de "Respuesta inmediata", si bien da una idea más clara de lo que se debe hacer, solo da la opción a reaccionar. Es decir, la solución por lo general será reactiva, porque dicha situación ya está presente en la organización.

- Las situaciones, sin embargo, que se ubican en el cuadrante "Anticipación", son más ideales, debido a que es posible llegar a ella con un plan hecho previamente y, para llegar a ella, lo ideal es antes haber pasado por el cuadrante llamado "Investigación".

- Las situaciones que se ubican en el cuadrante de "Problema/Oportunidad desperdiciada", se les llama así porque si bien están presentes

Fuente: Elaboración propia.

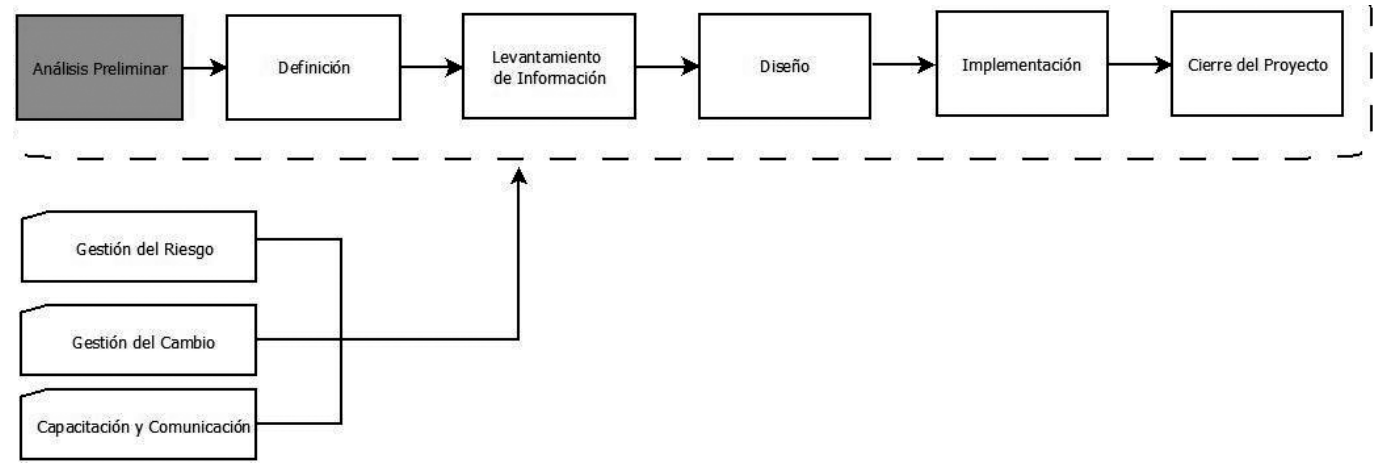

Figura 7. Ciclo de vida de un proyecto de BI propuesto. 
Fuente: Elaboración propia.

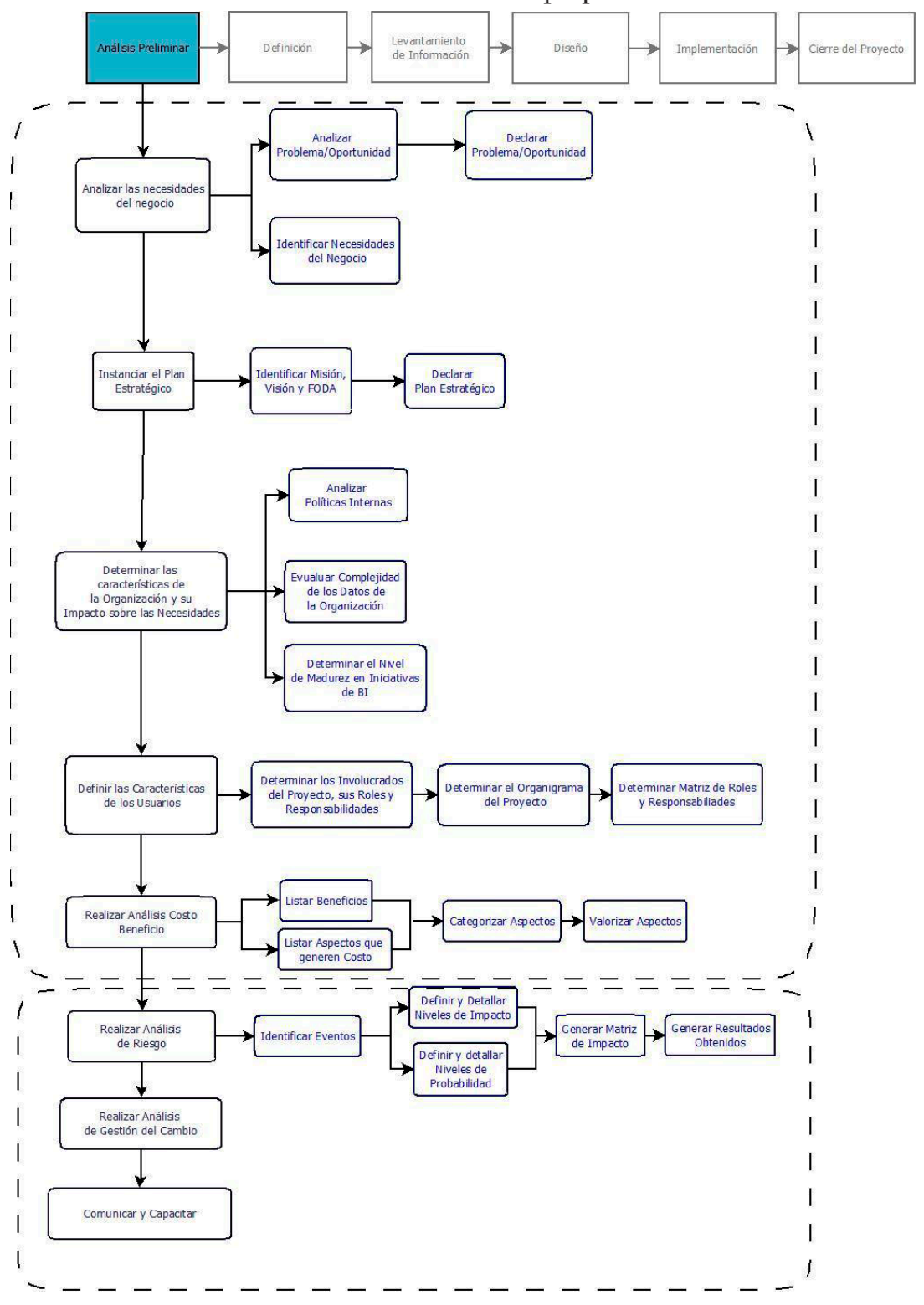

Figura 8. Detalle actividades específicas y transversales de la etapa preliminar.

en el tiempo, no se tiene claridad de qué las origina.

\section{CONCLUSIONES}

En este trabajo se ha presentado una propuesta de ciclo de vida de un proyecto de BI centrado en la etapa preliminar, que se alinea con un modelo de madurez organizacional. Esta alineación nace de la necesidad de buscar un modelo de madurez que pudiera ser utilizado para su aplicación como parte de una actividad dentro de dicha etapa. Para este fin, se revisaron trabajos relacionados con métodos de comparación entre modelos, sin embargo, no pudieron ser aplicados debido a que los modelos disponibles tienen distinto ámbito de cobertura y no se ajustaban a el propósito de este trabajo. Por lo tanto, se realizó un proceso de caracterización y evaluación de un conjunto de modelos de madurez $\mathrm{BI}$, con el fin de seleccionar objetivamente uno de 
Tabla 7. Descripción de actividades de la etapa de Análisis Preliminar.

\begin{tabular}{|c|c|c|c|}
\hline Actividades & Sub actividades & Objetivo & Riesgos de no realizar la actividad \\
\hline \multirow{3}{*}{$\begin{array}{l}\text { Identificar } \\
\text { Necesidades } \\
\text { del negocio }\end{array}$} & Análisis de problema/oportunidad & $\begin{array}{l}\text { Que los participantes claves Analicen, } \\
\text { definan y comprendan los problemas } \\
\text { u oportunidades de negocios de la } \\
\text { organización. }\end{array}$ & \multirow{3}{*}{$\begin{array}{l}\text { Repetir los errores que se cometen } \\
\text { actualmente. } \\
\text { Tener una visión sesgada de la situación } \\
\text { actual del negocio que no permita conocer } \\
\text { las necesidades y oportunidades que } \\
\text { sienten las bases para la formulación } \\
\text { de un proyecto sólido. }\end{array}$} \\
\hline & Declaración de Problema/Oportunidad. & $\begin{array}{l}\text { Que todos los participantes comprendan } \\
\text { y estén de acuerdo con cada una de las } \\
\text { descripciones. }\end{array}$ & \\
\hline & Identificar Estado actual del negocio & $\begin{array}{l}\text { Obtener conocimiento suficiente del } \\
\text { dominio del problema. }\end{array}$ & \\
\hline \multirow{2}{*}{$\begin{array}{l}\text { Instanciar } \\
\text { el plan } \\
\text { estratégico }\end{array}$} & $\begin{array}{l}\text { Identificar Misión y visión. } \\
\text { Identificar Valores. } \\
\text { Identificar FODA. }\end{array}$ & $\begin{array}{l}\text { Que los participantes conozcan y } \\
\text { comprendan cada uno de los componentes } \\
\text { del plan estratégico. }\end{array}$ & \multirow{2}{*}{$\begin{array}{l}\text { Desconocer la estrategia y objetivo } \\
\text { de negocio de la organización, lo que } \\
\text { propicia un desarrollo de proyecto no } \\
\text { alineado con el plan estratégico de la } \\
\text { organización. }\end{array}$} \\
\hline & Declaración del plan estratégico & $\begin{array}{l}\text { Documentar el plan estratégico que } \\
\text { servirá de lineamiento para el desarrollo } \\
\text { de proyectos. }\end{array}$ & \\
\hline \multirow{3}{*}{$\begin{array}{l}\text { Determinar } \\
\text { las } \\
\text { características } \\
\text { de la } \\
\text { organización } \\
\text { y su impacto } \\
\text { sobre las } \\
\text { necesidades }\end{array}$} & Análisis de las políticas internas & $\begin{array}{l}\text { Que los participantes identifiquen y } \\
\text { comprendan las políticas internas de la } \\
\text { organización. }\end{array}$ & $\begin{array}{l}\text { Desarrollo de un proyecto no legal, } \\
\text { ya que no se rige por las políticas } \\
\text { organizacionales. }\end{array}$ \\
\hline & $\begin{array}{l}\text { Evaluar complejidad de los datos de la } \\
\text { organización (alto nivel) }\end{array}$ & $\begin{array}{l}\text { Que los participantes identifiquen y } \\
\text { comprendan cómo obtienen y manipulan } \\
\text { los datos actualmente. }\end{array}$ & $\begin{array}{l}\text { Desarrollo de un proyecto que no aborde } \\
\text { mejoras en cuanto al manejo de la } \\
\text { información dentro de la organización }\end{array}$ \\
\hline & $\begin{array}{l}\text { Determinar el nivel de madurez en iniciativas } \\
\text { de BI }\end{array}$ & $\begin{array}{l}\text { Comprender los cambios que la orga- } \\
\text { nización necesita hacer para incrementar } \\
\text { el valor que BI. }\end{array}$ & $\begin{array}{l}\text { Desarrollo de un proyecto que tenga una } \\
\text { visión sesgada de la situación actual de la } \\
\text { organización, por lo tanto, no sea factible } \\
\text { su utilización ya que no se ajustará a las } \\
\text { necesidades de la organización. }\end{array}$ \\
\hline \multirow{3}{*}{\begin{tabular}{|l|} 
Definir las \\
características \\
de los \\
usuarios
\end{tabular}} & $\begin{array}{l}\text { Determinar a los involucrados del proyecto, } \\
\text { sus roles y responsabilidades }\end{array}$ & $\begin{array}{l}\text { Que los participantes identifiquen los } \\
\text { usuarios y sus roles. }\end{array}$ & $\begin{array}{l}\text { Resistencia por parte de los usuarios } \\
\text { que fueron omitidos por falta de } \\
\text { conocimiento. }\end{array}$ \\
\hline & Determinar el Organigrama del proyecto & $\begin{array}{l}\text { Que los participantes comprendan el } \\
\text { organigrama y su jerarquía. }\end{array}$ & $\begin{array}{l}\text { Visión sesgada de todos los posibles } \\
\text { involucrados en un proyecto a desarrollar. }\end{array}$ \\
\hline & Determinar Matriz de Roles y Responsabilidades & $\begin{array}{l}\text { Que los participantes comprendan } \\
\text { los roles y responsabilidades de cada } \\
\text { miembro involucrado. }\end{array}$ & $\begin{array}{l}\text { Desarrollo de un proyecto con visión } \\
\text { sesgada de la relación entre miembros del } \\
\text { equipo involucrados en dicho proyecto. }\end{array}$ \\
\hline \multirow{4}{*}{$\begin{array}{l}\text { Análisis } \\
\text { Costo- } \\
\text { beneficio }\end{array}$} & Realizar un listado de beneficios. & \multirow{4}{*}{$\begin{array}{l}\text { Determinar los costos y los bene- } \\
\text { ficios tangibles e intangibles. } \\
\text { Los resultados del análisis deben indicar } \\
\text { cómo la aplicación de BI podría resolver } \\
\text { un problema de negocio o habilitar una } \\
\text { oportunidad de negocio. }\end{array}$} & \multirow{4}{*}{$\begin{array}{l}\text { Desborde de presupuesto, rechazo y } \\
\text { fracaso de un proyecto que no contempla } \\
\text { el costo ni justifica su gasto. }\end{array}$} \\
\hline & $\begin{array}{l}\text { Realizar un listado de los aspectos que generan } \\
\text { algún costo. }\end{array}$ & & \\
\hline & $\begin{array}{l}\text { Categorizar cada aspecto de acuerdo al punto } \\
\text { de vista. }\end{array}$ & & \\
\hline & En base a la ponderación, valorar cada aspecto & & \\
\hline \multirow{5}{*}{$\begin{array}{l}\text { Análisis de } \\
\text { Riesgo }\end{array}$} & $\begin{array}{l}\text { Identificar los eventos que se encuentran } \\
\text { presentes, en términos de personas, habilidades, } \\
\text { procesos y tecnologías, basados en las } \\
\text { características de cada nivel de madurez de } \\
\text { Gartner (F1T3S3). }\end{array}$ & \multirow{5}{*}{$\begin{array}{l}\text { Evaluar en términos de personas, } \\
\text { habilidades, procesos y tecnologías los } \\
\text { riesgos asociados. } \\
\text { Se evaluará en base a lo determinado } \\
\text { por la aplicación del modelo de madurez } \\
\text { de Gartner. }\end{array}$} & \multirow{5}{*}{$\begin{array}{l}\text { Fracaso de un proyecto que no es capaz } \\
\text { de contemplar las distintas eventualidades } \\
\text { que puedan surgir antes, durante y } \\
\text { después del desarrollo de mismo. }\end{array}$} \\
\hline & $\begin{array}{l}\text { Definir y detallar los niveles de impacto a } \\
\text { utilizar para medir el riesgo }\end{array}$ & & \\
\hline & $\begin{array}{l}\text { Definir y detallar los niveles de probabilidad } \\
\text { de ocurrencia de un evento para medir el grado } \\
\text { de riesgo }\end{array}$ & & \\
\hline & $\begin{array}{l}\text { Generar la matriz de riesgo evaluando cada } \\
\text { evento con su impacto y probabilidad }\end{array}$ & & \\
\hline & Generar los resultados obtenidos & & \\
\hline
\end{tabular}

Fuente: Elaboración Propia. 


\begin{tabular}{|c|c|c|c|c|}
\hline \multirow[b]{2}{*}{ Tiempo } & & \multicolumn{2}{|c|}{ Problema/Oportunidad } & \\
\hline & & explicitos & no explicitos & \\
\hline \multirow{4}{*}{ Presentes } & \multirow{4}{*}{ urgente } & \multirow{4}{*}{ Respuesta inmediata } & \multirow{4}{*}{$\begin{array}{l}\text { Problema/Oportunidad } \\
\text { desperdiciada }\end{array}$} & evidente \\
\hline & & & & no evidente \\
\hline & & & & evidente \\
\hline & & & & no evidente \\
\hline \multirow{5}{*}{ Futuros } & \multirow{5}{*}{ No urgente } & \multirow{4}{*}{ Anticipación } & \multirow{4}{*}{ Investigación } & evidente \\
\hline & & & & no evidente \\
\hline & & & & evidente \\
\hline & & & & no evidente \\
\hline & & Conocida desconocida & \begin{tabular}{l|l|} 
conocida & desconocida \\
\end{tabular} & \\
\hline
\end{tabular}

Figura 9. Matriz Problema/Oportunidad de la sub-actividad Análisis de Problema/ Oportunidad.

ellos, a través de la alineación de las actividades que detalla el modelo de madurez analizado, con las actividades detalladas en un proyecto de BI, siendo elegido el modelo de madurez Gartner. El modelo Gartner fue seleccionado porque contenía la mayor cantidad de actividades concentradas en una etapa inicial del ciclo de vida de un proyecto de BI. Este modelo sirvió como base para la formulación del ciclo de vida propuesto de un proyecto de BI.

La caracterización del modelo de madurez y la alineación con las actividades del proyecto de BI, permitió comprender que el modelo MEI podría ser utilizado para el desarrollo de las siguientes etapas del ciclo de vida propuesto. Esto puede llevar a un trabajo futuro.

En lo relativo al ciclo de vida de un proyecto BI, la propuesta estuvo basada en una adaptación de lo propuesto en [17]. Esta propuesta incluye como una contribución una etapa de Análisis Preliminar, la cual es fundamental para establecer el proyecto en sintonía con la realidad y necesidad de la organización. Adicionalmente, la propuesta incluye para cada fase del ciclo la definición de tareas, sub-tareas, objetivos e identificación de riesgos asociados a la no realización de la tarea. También se incluye como parte del ciclo de vida propuesto la definición de activos de proceso para cada subactividad, donde se establece el cómo generar las salidas asociadas a ella.

La propuesta incluye también el análisis de riesgos asociados a las actividades y subactividades de cada etapa, permitiendo la claridad respecto a los riesgos involucrados en omitir o parcializar la realización de una actividad o subactividad. La identificación de riesgos está asociada a los niveles de madurez organizacional en qué riesgo podría presentarse. La importancia de la identificación de riesgos en proyectos de BI radica en que, por su naturaleza, muchas veces asociada al nivel estratégico de la organización, su no consideración puede llevar a la interrupción o la obtención de un bajo beneficio o impacto del mismo.

Actualmente se encuentra en desarrollo la aplicación de la propuesta a una unidad organizacional, a fin de contar con evidencia empírica que permita evaluar su grado de utilidad, nivel de completitud, y su eventual afinamiento y mejora de sus componentes $\mathrm{y}$ artefactos definidos.

\section{REFERENCIAS}

[1] P. Brooks, O. El-Gayar and S. Sarnikar, "Towards a Business Intelligence Maturity Model for Healthcare". 46th Hawaii International Conference on System Sciences. Hawaii. 2013.

[2] D. Cordero-Guzmán y G. Rodríguez-López. "La inteligencia de negocios: una estrategia para la gestión de las empresas productivas". Revista Ciencia UNEMI. Vol. 10 N $^{\circ} 43$, pp. 41-47. 2017.

[3] O. Montaño Arango, J.R. Corona Armenta y A.O. Ortega Reyes. "El Efecto de la Madurez de los Procesos Empresariales". XV Congreso Internacional de investigación en ciencias administrativas. Hidalgo. 2010.

[4] C.-S. Tan, Y.-W. Sim and W. Yeoh. "A Maturity Model of Enterprise Business Intelligence". 
Communications of the IBIMA. Vol. 2011. 2011.

[5] I. Hribar Rajteric. "Overview of Business Intelligence Maturity Models". 2010.

[6] E. Pérez Mergarejo, I. Pérez Vergara y Y. Rodríguez Ruiz. "Modelos de madurez y su idoneidad para aplicar en pequeñas y medianas empresas". Ingeniería Industrial. Vol. XXXV Nº 2, pp. 146-148. 2014.

[7] A. Fabián Duque y F. Salazar Pico. "Guía para la implementación del modelo de Gobierno para Business Intelligence". 2013. URL: https:// repositorio.espe.edu.ec/bitstream/21000/8899/2/ AC-MGS-ESPE-048286.pdf

[8] C.Min Hooi y W. Kee Luen. "Enterprise Business Intelligence Maturity Model: Case Study in Financial Industry". Journal of Southeast Asian Research. Vol. 2013, p. 17. 2013.

[9] A.D. Duncan and C. Howson. "Gartner Research". 2015. URL: https://www.gartner. com/en/documents/3136418

[10] M.Z. Torres Samaniego. "Estudio Comparativo entre los Estándares ISO/IEC TR 15504 y CMMI". Quito. 2007.

[11] R.D. Prieto Morales, C. Meneses Villega y V. Vega Zepeda. "Análisis comparativo de modelos de madurez en inteligencia de negocio". Ingeniare. Revista chilena de ingeniería. Vol. $23 \mathrm{~N}^{\circ}$ 3, pp. 361-371. 2015.

[12] S. Teruel. "Captio". 2014. URL: https://www.captio.net/BLOG/ EN-QUE-CONSISTE-UN-PROYECTODE-BUSINESS-INTELLIGENCE-BI

[13] J.R. Rodriguez. "Características de los proyectos de inteligencia de negocios". Catalunya. 2015.

[14] J.M. Pereira Vesga. "Desafíos en el desarrollo de iniciativas de inteligencia de negocios". Sistemas. 2015.

[15] P.M.I. (PMI). "Guía de los Fundamentos de la Dirección de Proyectos". 3ra Edición.

[16] I. Project Management Institute. "La guía de los fundamentos para la dirección de proyectos (Guía del PMBOK)". Sexta Edición. Pennsylvania. 2017.

[17] E. Buitrago. "Metodología de Desarrollo de Proyectos de Inteligencia de Negocios". p. 67.2004.

[18] L. Moss y S. Atre. "Business Intelligence Roadmap: The Complete Project Lifecycle for Decision-Support Applications". Addison Wesley. 2003. 